\title{
EXTRACCIÓN Y CARACTERIZACIÓN DE GLUCANO OBTENIDO DE LA VAINA DE LA MORINGA (MORINGA OLEIFERA)
}

\section{EXTRACTION AND CHARACTERIZATION OF GLUCAN OBTAINED FROM THE MORINGA POD (MORINGA OLEIFERA)}

\author{
${ }^{1 \star}$ Rincón E. Jessica P., Torres B. Darwin F., Rodríguez Q. Sandra P.
}

\begin{abstract}
${ }^{1}$ Universidad Francisco de Paula Santander. Facultad de Ciencias Agrarias y del Ambiente. Ingeniería Agroindustrial. Grupo de Investigación en Ciencia y Tecnología de Alimentos GICITECA. Semillero de Investigación en Aprovechamiento de Residuos y Bioprocesos Agroindustriales SINARBA. Cúcuta, Colombia. iessicapatriciare@gmail.com*,torres 18fabian@hotmail.com, sandrapatriciarq@ufps.edu.co
\end{abstract}

Recibido 21 de febrero de 2017; aceptado 30 de mayo de 2017.

RESUMEN

La Moringa es una de las plantas con mayor uso industrial en Norte de Santander. Su explotación ha generado una problemática ambiental por la aglomeración de vainas de degradación lenta en los campos posterior a la extracción y procesamiento de la semilla. Estudios han demostrada que la vaina de la planta se encuentra constituida por un polisacárido denominado Glucano perteneciente a los grupos de los $\beta$-Glucanos, sustancia susceptible a ser extraída y caracterizada mediante métodos fisicoquímicos de separación. Al caracterizar la vaina de Moringa producida en 
Autor a quien dirigir la correspondencia: Rincón Esteban Jessica Patricia. Correo electronico: jessicapatriciare@gmail.com

el municipio de Puerto Santander, se pudo establecer que está compuesta por un $57.39 \%$ de fibra, lo que determino que podría tener un alto contenido de Glucano. Se aplicaron tres métodos de extracción del compuesto por precipitación, hidrolisis acida y básica que se combinaron con los tamaños de partícula de $0.600,1.18$ y $2.36 \mathrm{~mm}$ en un diseño experimental Anova de dos factores con tres niveles, cada factor en una distribución de $3^{2}$. El glucano obtenido fue químicamente caracterizado mediante pruebas de Fehling, DNS, Molish y Lugol, esta última descarto la presencia de almidones y por lo tanto de a-glucano. Los datos se procesaron estadísticamente mediante el método de LSD de Fisher que determino que el mejor tratamiento combino el tamaño de partícula de $2.36 \mathrm{~mm}$ con el método básico. El Glucano obtenido fue sometido a pruebas de celulosa, FDA y FDN en las que se obtuvo $60.20 \%$ de Carboximetilcelulosa o $\beta$-Glucano denominada fibra soluble, $26.83 \%$ de fibra insoluble y $12.97 \%$ de compuestos secundarios.

Palabras Clave: Aprovechamiento, fibra dietética, pruebas fisicoquímicas, residuo.

\section{ABSTACT}

Moringa is one of the plants with greater industrial use in Norte de Santander. Its exploitation has generated an environmental problem due to the agglomeration of pods of slow degradation in the fields after the extraction and processing of the seed. Studies have shown that the pod of the plant is constituted by a polysaccharide called Glucan belonging to the groups of $\beta$-Glucans, substance susceptible to be extracted and characterized by physicochemical separation methods. When characterizing the Moringa pod produced in the municipality of Puerto Santander, it could be 
established that it is composed of $57.39 \%$ fiber, which determined that it could have a high Glucan content. Three methods of compound extraction were applied by precipitation, acid and basic hydrolysis that were combined with the particle sizes of $0.600,1.18$ and $2.36 \mathrm{~mm}$ in an experimental two-factor Anova design with three levels, each factor in a $3 \times 3$ distribution. The obtained glucan was chemically characterized by Fehling, DNS, Molish and Lugol tests, the latter ruling out the presence of starches and therefore $\alpha$-glucan. The data were processed statistically by Fisher's LSD method which determined that the best treatment combined the $2.36 \mathrm{~mm}$ particle size with the basic method. The Glucan obtained was subjected to cellulose, FDA and NDF tests in which $60.20 \%$ of Carboxymethylcellulose or $\beta$-Glucan was obtained, soluble fiber, $26.83 \%$ of insoluble fiber and $12.97 \%$ of secondary compounds.

Key words: Harvesting, dietary fiber, physicochemical tests, waste.

\section{INTRODUCCIÓN}

La contaminación ambiental constituye uno de los problemas más preocupantes para la sociedad, tema que hoy es motivo de discusión e investigación.

Los residuos sólidos son materiales de desecho que se incrementan exponencialmente mediante la implementación de procesos productivos; esto ha ocasionado un impacto ambiental negativo por la disposición inadecuada y la falta de uso de residuos en procesos alternativos, asunto que asociado al incremento de la población humana, a los procesos de transformación industrial y a los hábitos de consumo, deterioran aceleradamente el medio ambiente.

En Colombia se ha hecho popular la utilización de la Moringa como planta de aprovechamiento agroindustrial. De ella se 
emplean casi todas sus partes vegetativas en las que se incluyen hojas, tallo, raíces, fruto y semillas. Estas últimas, son las más utilizadas en la alimentación, la medicina, el tratamiento de aguas y la fertilización de cultivos (Foidl et al., 2001).

Pero el aprovechamiento de las semillas, genera residuos sólidos producidos por la acumulación de las vainas que envuelven la semilla.

Esta biomasa vegetal no presenta ningún uso específico y constituye un residuo de degradación lenta que tiende a convertirse en un foco de contaminación por su susceptibilidad a acumular fácilmente otras sustancias que en combinación con la vaina, producen malos olores y lixiviados que alteran las condiciones del suelo convirtiéndolas en agentes promotores de enfermedades en las zonas agrícolas productoras.

En la vereda Puerto Villamizar del municipio de Puerto Santander en el departamento de Norte de Santander, se cuenta con cultivos de Moringa empleados para el aprovechamiento en la transformación de diversos productos comerciales, haciendo especial énfasis en el uso de la semilla para la obtención de aceite, producción de harinas y elaboración de agentes coagulantes.
A medida que la semilla de Moringa es requerida para el desarrollo de diferentes productos, la vaina que la envuelve va aumentando como residuo acumulado en diversas zonas aledañas a los cultivos principales como se muestra en la figura 1 , situación que determina la necesidad de establecer procesos que permitan la disposición final y aprovechamiento de este residuo.

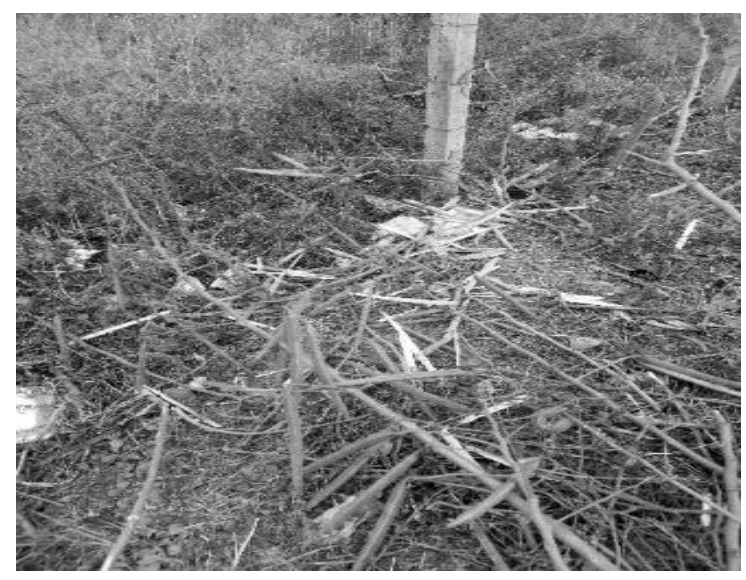

Figura 1. Acumulación de vainas de la moringa

Por otra parte, la vaina de la Moringa, es una biomasa vegetal constituida en un $32 \%$ por un compuesto conocido como glucano (Martín \& Puls, 2011). Esta sustancia posee usos diversos en la industria de la producción de alimentos y afines, pero carece de estudios que determinen los métodos y condiciones de su extracción.

Para determinar el método de extracción más adecuado para la obtención del 
glucano de la vaina de la Moringa y caracterizarlo para establecer sus usos industriales, se llevó a cabo la presente investigación que buscó adoptar una alternativa de solución que permita reutilizar este desecho y así contribuir a la reducción de la contaminación medioambiental producida por la acumulación de residuos orgánicos.

\section{MATERIALES Y MÉTODOS}

El presente estudio se enmarca en una investigación cuasi-experimental en la que se obtuvo una muestra de $10 \mathrm{~kg}$ de vainas de 5 hectáreas cultivadas de plantas de Moringa.

La vaina fue recolectada, seleccionada y clasificada de acuerdo a parámetros de color, forma, tamaño y daños externos para luego ser almacenadas en bolsas de polietileno.

Las vainas fueron sometidas a un proceso de reducción de tamaño empleando un molino de martillos de marca PS\&E modelo MM12 y fueron clasificadas en un vibrotamiz de marca Fritsch modelo Analysette 18 para separar los tamaños de partícula específicos de $0.600,1.18$ y $2.36 \mathrm{~mm}$ que se combinaron con los métodos de extracción ácido, básico y precipitado que dieron origen a los nueve tratamientos que se plantean en la tabla 1.

El material molido y clasificado fue llevado al equipo de extracción sólido-líquido por lixiviación con el fin de realizar la inactivación de la enzima betaglucanasa presente en la composición de la vaina y que causa la despolimerización del betaglucano. Este proceso se llevó a cabo con una mezcla de agua-alcohol en una relación $1 / 4$, en la que el alcohol asciende por la columna de extracción condensa y lixivia sobre el sólido arrastrando las betaglucanasas, procedimiento que se realizó a reflujo total y de forma continua por un tiempo de dos horas.

Tabla 1. Tratamientos planteados

\begin{tabular}{|c|c|c|}
\hline Tratamientos & $\begin{array}{l}\text { Métodos } \\
\text { de } \\
\text { extracción }\end{array}$ & $\begin{array}{l}\text { Tamaño de } \\
\text { partícula } \\
(\mathrm{mm})\end{array}$ \\
\hline $\mathrm{T} 1$ & \multirow{3}{*}{ Precipitado } & 0,600 \\
\hline T2 & & 1,18 \\
\hline T3 & & 2,36 \\
\hline $\mathrm{T} 4$ & \multirow{3}{*}{ Acido } & 0,600 \\
\hline T5 & & 1,18 \\
\hline T6 & & 2,36 \\
\hline $\mathrm{T7}$ & \multirow{3}{*}{ Básico } & 0,600 \\
\hline T8 & & 1,18 \\
\hline T9 & & 2,36 \\
\hline
\end{tabular}

El material obtenido fue filtrado para separar la parte líquida que fue desechada, mientras 
que el sólido fue llevado a un secado convectivo en un secador de charolas a una temperatura de $100^{\circ} \mathrm{C}$.

El material seco de cada uno de los tamaños de partícula, fue dividido para la aplicación de los tres métodos de extracción donde en el método básico se empleó hidróxido de sodio a una concentración 0,4 molar, que se llevó a una temperatura de $80^{\circ} \mathrm{C}$ en agitación constante por un tiempo de dos horas. En el método ácido se empleó ácido sulfúrico a una concentración de 0,9 molar que se llevó a una temperatura de $80^{\circ} \mathrm{C}$ en agitación constante por un tiempo de 15 minutos, posteriormente el material fue lavado $y$ llevado a recalentamiento a $57^{\circ} \mathrm{C}$ en agua por un tiempo de dos horas. En el método de precipitado se empleó agua y una muestra de vaina que se llevó a una temperatura de $57-60{ }^{\circ} \mathrm{C}$ en agitación contante por un periodo de 3 horas.
El producto obtenido de los métodos ácido y básico fue lavado para corregir el $\mathrm{pH}$ y posteriormente fue filtrado al igual que el producto obtenido del método precipitado.

El material sólido separado en los tres métodos propuestos fue llevado a un proceso de congelación a una temperatura de -18 a $-20^{\circ} \mathrm{C}$ por un tiempo de 48 horas con la finalidad de extraer parte del agua de composición por rompimiento de la membrana celular.

Transcurrido este tiempo el material fue descongelado sobre papel filtro y llevado ha secado convectivo a una temperatura de 60 ${ }^{\circ} \mathrm{C}$ por dos horas.

\section{RESULTADOS Y DISCUSIÓN}

La materia prima utilizada que se muestra en la figura 2, presentó una forma triangular, con unas medidas promedio de $31 \mathrm{~cm}$ de largo por $2 \mathrm{~cm}$ de ancho, con un peso medio de 7,45 g/vaina, con un contenido de 12 a 20 semillas por fruto.

La composición química de la vaina de acuerdo a lo que se muestra en la tabla 2, establece que posee un $1.76 \%$ de extracto etéreo lo cual indica que esta materia prima no es una fuente propia para la extracción de aceites, un contenido proteico que alcanzo un $6.2 \%$, un porcentaje representativo de cenizas con $5.8 \%$ y un contenido de humedad que ascendió al $11,12 \%$. 


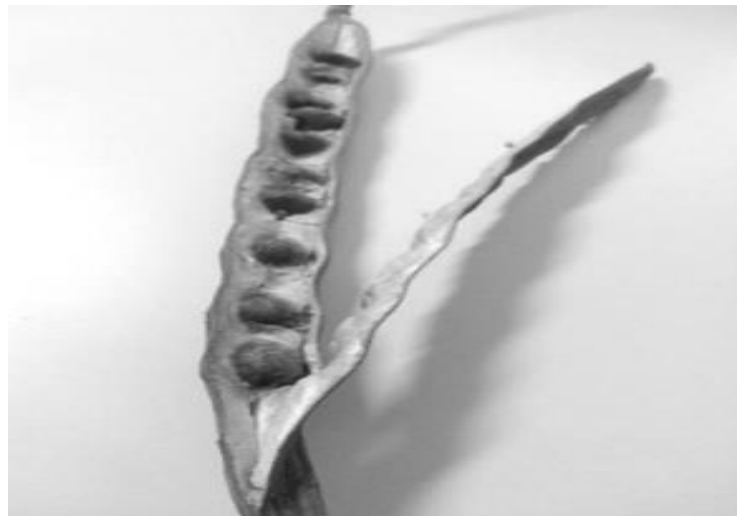

Figura 2. Fruto de la moringa

Para la extracción de glucano era importante determinar el porcentaje de fibra cruda en la que se obtuvo un $57.39 \%$, resultado que al ser comparado con lo obtenido por (García, Martínez, \& Rodríguez, 2013) que registraron un $4.8 \%$ de contenido de fibra cruda en vainas de moringa cultivadas en San Salvador. Frente a esto se determinó una diferencia importante, siendo la vaina de moringa cultivada en Puerto Santander rica en fibra y por ende ideal para el presente estudio.

Con respecto a la cantidad de carbohidratos, se logró establecer que esta asciende a un 17,73\%. Este resultado, en comparación con la investigación desarrollada por Liñán (Liñán Tobias, 2010) que alcanzó un contenido de carbohidratos del $14,3 \%$ en vainas de moringa provenientes de la ciudad de Cartagena, es superior.
Estos factores indicaron que la vaina de moringa producida en el municipio de Puerto Santander es rica en fibra $y$ azucares; siendo esto valores significativos para el desarrollo de la investigación. En la tabla 2 se estableció la caracterización química completa de la vaina de moringa estudiada.

Tabla 2. Caracterización química de la vaina de la moringa

\begin{tabular}{|l|c|}
\hline \multicolumn{1}{|c|}{ Prueba } & $\begin{array}{c}\text { Resultados } \\
\text { obtenidos }\end{array}$ \\
\hline $\begin{array}{l}\text { Extracto } \\
\text { etéreo }\end{array}$ & $1.76 \pm 0.07$ \\
\hline Proteínas & 6.2 \\
\hline Cenizas & $5.8 \pm 0.22$ \\
\hline Humedad & $11.12 \pm 0.11$ \\
\hline Fibra cruda & $57.39 \pm 0.92$ \\
\hline Carbohidratos & 17.73 \\
\hline
\end{tabular}

Nota. $\mathrm{n}=2$, media \pm desviación típica.

En la tabla 3, se muestra la cantidad de glucano obtenido después de las extracciones. En ella se puede evidenciar que el mejor tratamiento de extracción es el básico dado que se obtiene la mayor cantidad de glucano. Aun cuando se plantearon tres tamaños de partícula, en cada uno de ellos, el tratamiento básico arrojo los mejores resultados en comparación a los mismos tamaños de partícula evaluados por los otros métodos. 
Tabla 3. Cantidad de glucano obtenido en cada extracción

\begin{tabular}{|c|c|}
\hline Tratamientos & $\begin{array}{c}\text { Resultados } \\
\text { obtenidos } \\
\text { (gramos) }\end{array}$ \\
\hline T1 & $0,1590 \pm 0,074$ \\
\hline T2 & $0,0685 \pm 0,015$ \\
\hline T3 & $0,4483 \pm 0,182$ \\
\hline T4 & $0,2485 \pm 0,147$ \\
\hline T5 & $0,5656 \pm 0,086$ \\
\hline T6 & $0,6001 \pm 0,164$ \\
\hline T7 & $0,3727 \pm 0,113$ \\
\hline T8 & $0,401 \pm 0,073$ \\
\hline T9 & $0,8706 \pm 0,117$ \\
\hline
\end{tabular}

Nota. $\mathrm{n}=3$, media \pm desviación típica.

Sin embargo de los tres tamaños de partícula propuestos el diámetro de 2.36 $\mathrm{mm}$ en el método básico denominado tratamiento T9, fue el que presentó una mayor cantidad de glucano extraído en cifras que alcanzaron una media de 0,8706 $g$ en estado seco, estableciéndose que de $100 \mathrm{~g}$ de muestra se obtiene $870.6 \mathrm{mg}$ de glucano. Este glucano se caracterizó mediante las pruebas químicas que se muestran en la tabla 4.

Estas pruebas se aplicaron a los nueve tratamientos planteados, donde la prueba de Fehling dio positivo en todos los casos para indicar la presencia de azucares reductores con la formación de un residuo de color rojo ladrillo, reacción de tipo oxidoreducción, en la que el azúcar reductor se oxida a ácido y el $\mathrm{Cu}$ II se reduce a $\mathrm{Cu} I$ (Velurtas, 2016).

Tabla 4. Caracterización del extracto

\begin{tabular}{|c|c|c|c|}
\hline \multirow{2}{*}{ Trat. } & \multicolumn{3}{|c|}{ Prueba } \\
\cline { 2 - 4 } & Fehling & Molish & Lugol \\
\hline T1 & + & + & - \\
\hline T2 & + & + & - \\
\hline T3 & + & + & - \\
\hline T4 & + & + & - \\
\hline T5 & + & + & - \\
\hline T6 & + & + & - \\
\hline T7 & + & + & - \\
\hline T8 & + & + & - \\
\hline T9 & + & + & - \\
\hline
\end{tabular}

La prueba de Molish detectó la presencia de hidratos de carbono en general, basándose en la acción hidrolizante y deshidratante del compuesto en presencia de ácido sulfúrico para formar sustancias complejas coloreadas (Delgado Fajardo, 2008). De acuerdo a ello, en cada uno de los tratamientos, se obtuvieron resultados favorables dado que en todos se formó un anillo de color violeta en la interface. Por esa razón se establece que la prueba Fehling fue positiva para carbohidratos.

Por otra parte y de acuerdo a la información recopilada, el grupo de los a-glucanos son compuestos caracterizados por poseer en su composición almidón. Por esa razón se realizaron pruebas de Lugol para determinar la presencia de almidones en los extractos obtenidos. Ninguno de ellos presentó viraje de color azul o rojo que indicaran la 
presencia de almidones o glucógeno respectivamente, siendo resultados oportunos en el presente estudio y de acuerdo a ello se descarta que los biocompuesto extraídos hagan parte del grupo de los a-glucanos.

Debido a que se determinó la presencia de azucares reductores en todos los tratamientos, estos fueron cuantificados empleando el método DNS que se muestra en la tabla 5, en la que se estableció que los tratamientos T4, T3 y T1, se destacan por presentar la mayor concentración de azúcares reductores.

Los resultados obtenidos mediante el método DNS se compararon con otros estudios, donde (Hurtado \& Suarez, 2016) determinaron la cantidad de beta-glucano en ciertos cereales que fueron sometidos a una previa extracción y a un pre-tratamiento con ultrasonido. De manera que cuantificaron los azúcares reductores por el método de Dubois; de ahí se lograron concentraciones de $0,0945854 \mu / \mathrm{ml}$ en la cebada, 0,07348221 $\mu / \mathrm{ml}$ en el arroz y $0,0047656 \mu / \mathrm{ml}$ para el trigo, valores muy inferiores a los obtenidos en la cuantificación de azúcares reductores realizada por el método DNS del glucano de la vaina de la moringa.
Tabla 5. Concentración de azúcares reductores presentes en los extractos

\begin{tabular}{|c|c|c|}
\hline Trat & $\begin{array}{c}\text { Absorbancia } \\
\text { (nm) }\end{array}$ & $\begin{array}{c}\text { Concentración } \\
\text { glucosa (g/L) }\end{array}$ \\
\hline T1 & 0,258 & 0,1126689 \\
\hline T2 & 0,234 & 0,1049778 \\
\hline T3 & 0,297 & 0,1251669 \\
\hline T4 & 0,384 & 0,1530471 \\
\hline T5 & 0,242 & 0,1075415 \\
\hline T6 & 0,185 & 0,0892752 \\
\hline T7 & 0,002 & 0,0306308 \\
\hline T8 & 0,253 & 0,1110666 \\
\hline T9 & 0,245 & 0,1085029 \\
\hline
\end{tabular}

Nota. $\mathrm{n}=3$, media \pm desviación típica.

Los resultados fueron analizados mediante una Anova de dos factores con tres niveles en cada uno de ellos, usando la prueba LSD de Fisher en una distribución experimental de $3^{2}$ con la ayuda del paquete estadístico Statgraphics, que determinó si esas diferencias eran estadísticamente significativas. Inicialmente se evaluó la cantidad de glucano extraído frente al tamaño de partícula de acuerdo a lo que se muestra en la tabla 6.

Tabla 6. Análisis de varianza para cantidad extraída y tamaño de partícula-suma de cuadrados tipo III 


\begin{tabular}{|c|c|c|c|c|c|}
\hline Fuente & $\begin{array}{c}\text { Sumade } \\
\text { Cuadrados }\end{array}$ & GI & $\begin{array}{c}\text { Cuadrado } \\
\text { Medio }\end{array}$ & $\begin{array}{c}\text { Razón } \\
\mathbf{F}\end{array}$ & $\begin{array}{c}\text { Valor- } \\
\mathbf{P}\end{array}$ \\
\hline $\begin{array}{c}\text { Efectos } \\
\text { principales }\end{array}$ & & & & & \\
\hline A.método & 0,7144 & 2 & 0,357223 & 8,71 & 0,0014 \\
\hline Residuos & 0,9837 & 24 & 0,0409914 & & \\
\hline $\begin{array}{c}\text { Total } \\
\text { corregido }\end{array}$ & 1,6982 & 26 & & & \\
\hline
\end{tabular}

En la tabla 6, se registra un valor $p$ de 0,0014 , lo que indica que hay diferencias estadísticamente significativas con valores de $(p<0,05)$ entre los tres tamaños de partícula evaluados.

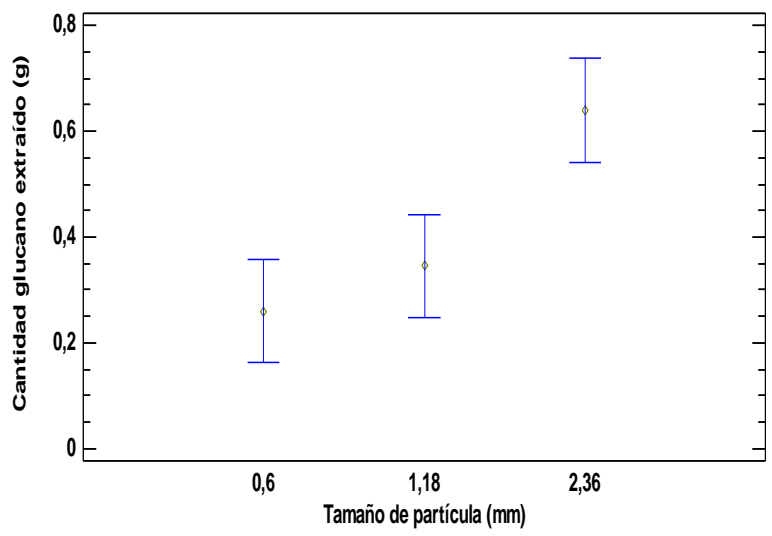

Figura 3. Medias y 95,0 de Fisher LSD cantidad extraída vs tamaño de partícula

Por consiguiente en la figura 3 , se muestra que el tamaño de partícula de 0.600 y 1.18 $\mathrm{mm}$ se obtuvo una menor cantidad de glucano extraído en comparación a lo alcanzado por el diámetro de $2.36 \mathrm{~mm}$ que presenta la diferencia significativa. Por lo tanto el mejor tamaño de partícula para la extracción de glucano de la vaina de la moringa lo representa el tamaño de 2.36 $\mathrm{mm}$.

Así mismo se evaluó la cantidad de glucano extraído frente a los métodos de extracción que se muestra en la tabla 7.

Tabla 7. Análisis de varianza para cantidad extraída y método de extracción-suma de cuadrados tipo III

\begin{tabular}{|c|c|c|c|c|c|}
\hline Fuente & $\begin{array}{c}\text { Sumade } \\
\text { Cuadrados }\end{array}$ & GI & $\begin{array}{c}\text { Cuadrado } \\
\text { Medio }\end{array}$ & $\begin{array}{c}\text { Razón- } \\
\text { F }\end{array}$ & $\begin{array}{c}\text { Valor- } \\
\mathbf{P}\end{array}$ \\
\hline $\begin{array}{c}\text { Efectos } \\
\text { principales }\end{array}$ & & & & & \\
\hline A:método & 0,512111 & 2 & 0,256055 & 5,18 & 0,0135 \\
\hline Residuos & 1,18613 & 24 & 0,049422 & & \\
\hline $\begin{array}{c}\text { Total } \\
\text { corregido }\end{array}$ & 1,69824 & 26 & & & \\
\hline
\end{tabular}

En la tabla 7, se registra un valor $p$ de 0,0135 lo cual indica que hay diferencias estadísticamente significativas $(p<0,05)$ entre los tres métodos de extracción empleados.

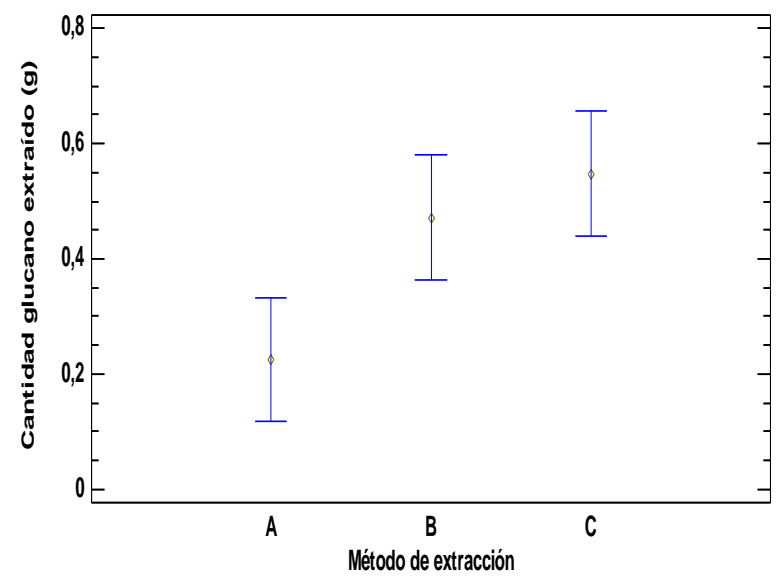


Figura 4. Medias y 95,0 de Fisher LSD cantidad extraída vs método de extracción

En la figura 4, se observa que el método de precipitado (A) presenta una cantidad de glucano extraído muy inferior en comparación a lo alcanzado por el método acido (B) y el método básico (C) que registran una mayor cantidad de glucano, y es por ello que dentro de estos dos últimos métodos de extracción se encuentra el mejor, aun cuando no hay diferencias estadísticamente significativas entre estos dos últimos, situación que hizo necesario evaluar otros aspectos como: rentabilidad económica e impacto ambiental.

En consecuencia se estableció que el valor comercial del $\mathrm{H}_{2} \mathrm{SO}_{4}$ es superior y en términos ambientales se caracteriza por ser una sustancia bastante agresiva y corrosiva que daña de forma inmediata todo aquello que entra en contacto con él. Mientras que el $\mathrm{NaOH}$ es más económico y al entrar en contacto con el medio ambiente tiende a cambiar sus características al ceder aniones y cationes, aspecto que permite cambiar su comportamiento químico y de esta manera disminuir el impacto ambiental generado.
Finalmente se concluye que el mejor tratamiento fue el T9 que corresponde al tamaño de partícula de $2.36 \mathrm{~mm}$ y al método básico. Los análisis planteados en la tabla 8 , solo fueron realizados al mejor tratamiento con el fin de establecer la composición química del $\beta$-glucano.

En la prueba de celulosa modificada se confirma la presencia de $\beta$-glucano y se cuantifica su contenido representado a la fibra soluble. Las pruebas de fibra en detergente ácido y fibra en detergente neutro permitieron cuantificar el restante de la composición química del $\beta$-glucano que comprende a la lignina, hemicelulosa y componentes minoritarios representando la fibra insoluble.

Tabla 8. Composición química del $\beta$-glucano

\begin{tabular}{|c|c|}
\hline Prueba & $\begin{array}{c}\text { Resultados } \\
\text { obtenidos (\%) }\end{array}$ \\
\hline $\begin{array}{c}\text { Celulosa } \\
\text { modificada }\end{array}$ & 60,20 \\
\hline FDA & 77,65 \\
\hline FDN & 87,03 \\
\hline Hemicelulosa & 9,38 \\
\hline Lignina & 17,45 \\
\hline $\begin{array}{c}\text { Cenizas, } \\
\text { nitrógeno y } \\
\text { minerales }\end{array}$ & 12,97 \\
\hline
\end{tabular}




\section{CONCLUSIONES}

Las vainas de la moringa producidas en el municipio de Puerto Santander presentaron un color marrón claro homogéneo, de forma triangular, con una longitud de $31 \mathrm{~cm}$ de largo por $2 \mathrm{~cm}$ de ancho y un peso por vaina de 7,45 g constituida por 10 a 12 semillas. Su composición química está determinada por un $57,39 \%$ de fibra cruda y $17,73 \%$ de carbohidratos, lo cual indica que es un residuo con gran aprovechamiento agroindustrial para la extracción de $\beta$ glucano por su elevado valor de polímeros de glucosa y azucares. Sin embargo este subproducto presento un $11,12 \%$ de humedad que indica un porcentaje representativo de agua, además la compone $6,2 \%$ de proteínas y 5,8 \% de cenizas valores que señalan la cantidad de aminoácidos esenciales y minerales de diversa naturaleza. Así mismo se concluye que la vaina no es una fuente de ácidos grasos con $1,76 \%$ de extracto etéreo.

Mediante la evaluación de los tres métodos de extracción se logró determinar que el mayor rendimiento se obtuvo en el tratamiento nueve que combina la variable de tamaño de partícula 2,36 $\mathrm{mm}$ e implementa el método básico. Los métodos restantes también evaluados en función del rendimiento arrojado en la extracción, presentan niveles inferiores del compuesto extraído aun cuando todos resultaron efectivos en la obtención de glucano.

Las características fisicoquímicas del glucano mediante las pruebas de identificación como lo son Fehling, Molish y la prueba de Lugol permitieron determinar la presencia de azúcares en los compuestos extraídos en todos los nueve tratamientos planteados, además que el compuesto presentaba las características de un carbohidrato y que no pertenecía al grupo de los almidones. Así mismo por el método DNS se determinó que en todos los métodos planteados se lograron concentraciones de azúcares reductores que no presentaron diferencias significativas entre sí.

Los análisis de fibra en detergente neutro y fibra en detergente ácido permitieron determinar la cantidad de fibra soluble e insoluble que comprende la fibra dietética, en lo que se obtuvo como resultado un $60,20 \%$ de $\mathrm{FS}, 26,83 \%$ de $\mathrm{FI}$ asimismo el $12,97 \%$ corresponde a otros compuestos como cenizas, nitrógeno y minerales, concluyendo con esto el $100 \%$ de la composición química del $\beta$-glucano. 
Del método básico se extrajo un 60,20 \% de celulosa modificada específicamente carboximetilcelulosa (CMC) del tipo $\beta-1,4-$ glucano clasificada como fibra dietética soluble, con lo cual se confirma la presencia del mismo en la vaina de la moringa recolectada en el municipio de Puerto Santander del departamento Norte de Santander, sustancia utilizada en la industria de alimentos como agente espesante y sustituto de grasas además siendo altamente beneficioso para la salud humana dado que disminuye los niveles de colesterol.

Mediante el análisis estadístico se comprueba la hipótesis alternativa, dado que se logró la separación, obtención, cuantificación y caracterización del betaglucano de la vaina de la moringa. Lo que permitió determinar que puede ser empleado en la realización de procesos químicos e industrial para la transformación y suplementación de alimentos, producción de aditivos, en la alimentación animal y en la industria farmacéutica en la elaboración de piezas de osteosíntesis, apósito para heridas y polimembranas entre otras.

\section{BIBLIOGRAFÍA}

Bohada Lizcano, Y. K., \& Figueroa Torres, D. F. (2017). Efecto del ultrasonido en la extracción del aceite de la semilla de moringa (Moringa oleífera) destinado para la producción de biodiesel en el municipio de Los Patios, Norte de Santander. Cúcuta: Universidad Francisco de Paula Santander.

Delgado Fajardo, B. I. (2008). Reacciones de los carbohidratos. Obtenido de Reacción de Molish: http://aprendeenlinea.udea.edu.co/lms/mo odle/pluginfile.php/45190/mod_resource/c ontent/0/reacciones_de_los_carbohidratos -2008.pdf

Foidl, N., Makkar, H. P. S., Becker, K., Foild, N., \& Km, S. (2001). the Potential of Moringa Oleifera for Agricultural and Industrial Uses. What Development Potential for Moringa Products?, 1-20.

Hurtado, Y., \& Suarez, Y. (2016). Evaluar el efecto del ultrasonido sobre la extracción 
del beta-glucano de cereales (cebada, trigo y arroz). San José de Cúcuta: Universidad Francisco de Paula Santander.

Jaramillo Henao, G., \& Zapata Márquez, L. M. (2008). Aprovechamiento de los residuos sólidos orgánicos en Colombia. Medellín: Universidad de Antioquia.

Liñán Tobias , F. (2010). Moringa Oleífera El árbol de la nutrición. Ciencia y salud virtual, 130-138.

Martín, C., \& Puls, J. (2011). Chemical characterisation of husks and fruit residues of J. curcas and M.oleifera (inédito).

Velurtas, S. M. (21 de Marzo de 2016). Introducción a la biología. Recuperado el 29 de Octubre de 2017, de Estudio y reconocimiento de las biomoleculas que forman parte de los organismos vivientes: https://www.researchgate.net/publication/2 67689885_Estudio_y_reconocimiento_de_ las_biomoleculas_que_forman_parte_de_I os_organismos_vivientes 